\title{
Reconfigurable Filter-free Sinc-shaped RF Photonic Filters Based on Rectangular Optical Frequency Comb
}

\author{
Jianqi Hu ${ }^{1}$, Simon J. Fabbri ${ }^{1, *}$, Camille-Sophie Brès ${ }^{1}$ \\ ${ }^{\text {I}}$ Ecole Polytechnique Fédérale de Lausanne, Photonic Systems Laboratory (PHOSL), STI-IEL, Station 11, CH-1015 Lausanne, Switzerland \\ *simon.fabbri@epfl.ch
}

\begin{abstract}
We demonstrate reconfigurable sinc-shaped RF photonic filters based on rectangular optical frequency comb synthesized from cascaded modulators. Simplicity of the approach and flexibility in bandwidth for fixed free-spectral-range is shown. Phase response is also investigated. OCIS codes: (060.5625) Radio frequency photonics; (120.2440) Filters; (230.2090) Electro-optical devices
\end{abstract}

\section{Introduction}

Radio frequency (RF) filters based on optical frequency comb and dispersive propagation enable RF signal processing in the photonic domain $[1,2]$. Compared to its electrical counterpart, this approach is favored due to the broad bandwidth and low losses rendered by optical fibers, as well as arbitrary RF filter shape empowered by lineby-line shaping of the optical frequency comb $[2,3]$. For instance, highly selective rectangular RF filter shape has been demonstrated using programmable pulse shapers to tailor optical frequency comb [4] or slice ASE source [5]. From a communication perspective, sinc-shaped RF photonic filters may offer great potential to high-speed orthogonal frequency division multiplexing (OFDM), optimizing the spectral efficiency among electrical, optical, and wireless applications. Given the complex features of sinc-shaped filter, direct implementation in the electrical domain is challenging. In this paper, we present reconfigurable RF photonic filters with near perfect sinc response based on a simple versatile comb source. The comb is synthesized using a cascade of Mach-Zehnder modulators (MZMs) driven by synchronized RF frequencies [6], resulting in rectangular spectra without the need for spectral shaping. The proposed RF filter scheme can be easily reconfigured by modifying the RF signals (frequency, power) and DC bias driving the comb source modulators. The flexibility of the proposed RF filter is here demonstrated by varying the number of comb lines, thereby the number of filter taps, while keeping the repetition rate constant and a near perfect sinc filter shape.

\section{Principle and experiment results}

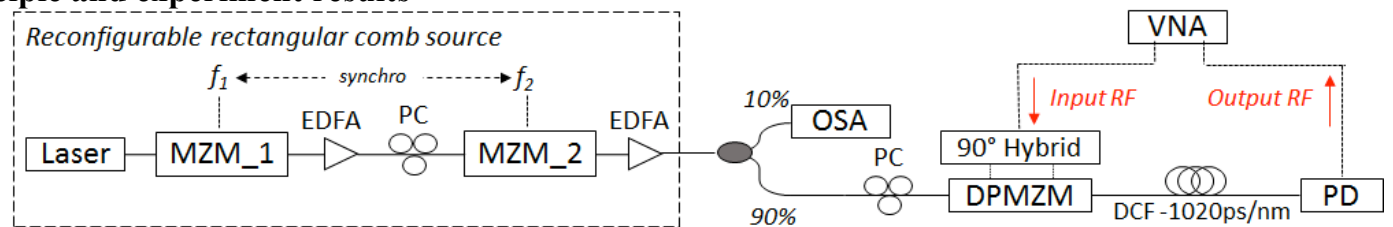

Fig. 1. Experiment setup of sinc-shaped RF photonic filter. MZM: Mach-Zehnder modulator, PC: polarization controller, DPMZM: dual-parallel Mach-Zehnder modulator, DCF: dispersion compensating fiber, VNA: vector network analyzer, PD: photodetector.

The experimental setup is depicted in Fig. 1. It consists in a comb source (dashed box) generated by electro-optic modulation of a CW laser using two cascaded MZMs, driven at frequency $f_{1}$ and $f_{2}$, respectively. The comb is then directed to a dual-parallel Mach-Zehnder modulator (DPMZM) through a polarization controller (PC). A small portion is tapped for spectrum monitoring on an optical spectrum analyzer (OSA). The RF signal is loaded to the inphase and quadrature ports of the DPMZM through a 90-degree hybrid coupler. The comb carriers and generated single-sidebands then propagate through a spool of dispersion compensating fiber (DCF) to induce differential group velocity delay, and finally detected by a photodetector (PD) to retrieve the filtered RF signal.

For a frequency comb of rectangular shape, the corresponding RF photonic filter response with respect to the first comb line can be expressed as $[7,8]$ :

$$
H\left(f_{R F}\right) \propto e^{i \varphi_{2} 2 \pi^{2} f_{R F}^{2}} \sum_{k=0}^{N-1} e^{i \varphi_{2} 4 \pi^{2} k f_{m} f_{R F}}=e^{i \varphi_{2} 2 \pi^{2} f_{R F}^{2}+i \varphi_{2} 2 \pi^{2}(N-1) f_{m} f_{R F}} \frac{\sin \left(\varphi_{2} 2 \pi^{2} N f_{m} f_{R F}\right)}{\sin \left(\varphi_{2} 2 \pi^{2} f_{m} f_{R F}\right)}
$$

where $N$ is the number of comb lines, $f_{R F}$ and $f_{m}$ are the RF frequency and comb repetition rate, respectively. $\varphi_{2}(-$ $1020 \mathrm{ps} / \mathrm{nm}$ in our experiment) stands for dispersion induced group delay. The term $\sin \left(\varphi_{2} 2 \pi^{2} N f_{m} f_{R F}\right) / \sin \left(\varphi_{2} 2 \pi^{2} f_{m} f_{R F}\right)$ is a periodic sinc function [6] with $(N-1)$ nulls equally distributed between the main peaks. The quadratic phase term represents a linear group delay additional to the periodic sinc carver, while the linear phase term vanishes, when the laser frequency is set as reference. 

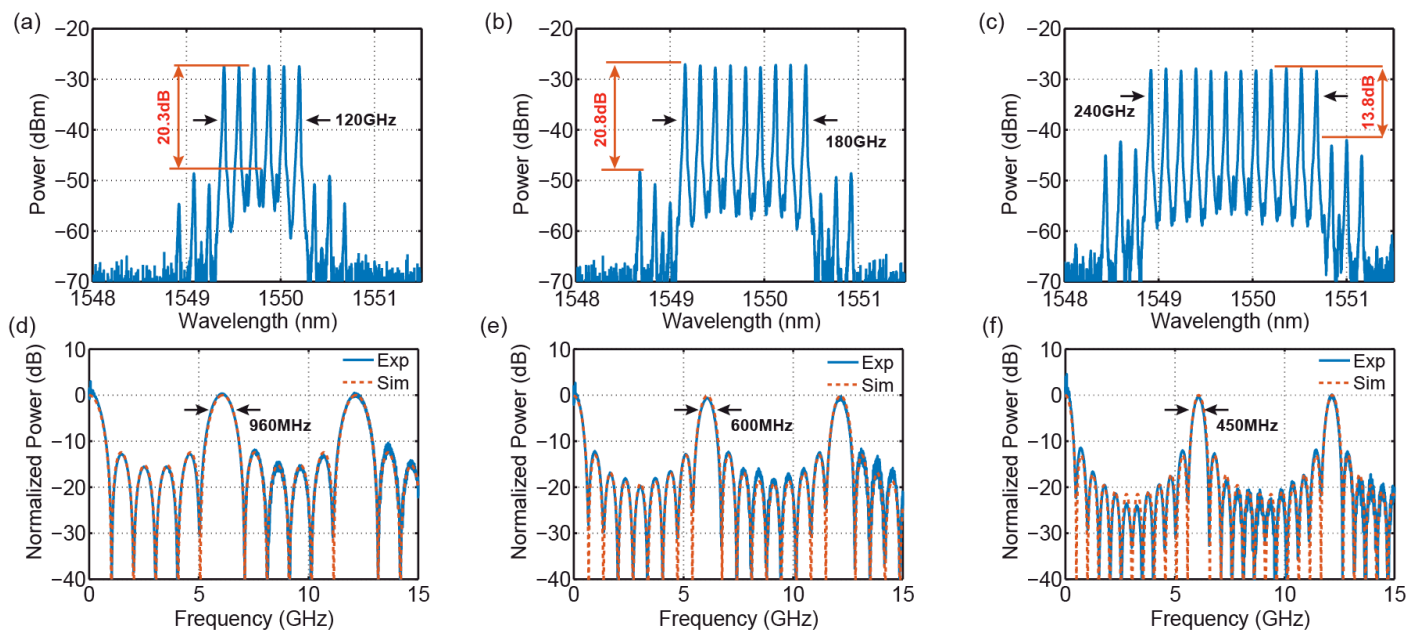

Fig. 2. (a) 6-line (b) 9-line (c) 12-line rectangular optical frequency comb at $20 \mathrm{GHz}$ repetition rate. Corresponding (d) 6-line (e) 9-line (f) 12-line frequency comb based sinc-shaped RF photonic filter response. Blue: experiment; Orange: simulation (ideal sinc).

For our demonstration, MZM_1 (low $\left.\mathrm{V}_{\pi}\right)$ is driven at $f_{l}=30 \mathrm{GHz}$. By properly setting the RF power and DC bias to MZM_1, 2-line (carrier suppressed), 3-line (odd-order harmonics suppressed), or 4-line (even-order harmonics suppressed) comb spaced at $2 f_{l}=60 \mathrm{GHz}$ are generated. MZM_2 is driven at $f_{2}=20 \mathrm{GHz}$ to triple the number of comb lines and lower the comb repetition rate to $f_{m}=f_{2}$. The resulting frequency combs with 6,9 , and 12 lines (Fig. 2. (a), (b), and (c)), have a side-mode suppression ratio of $20.3 \mathrm{~dB}, 20.8 \mathrm{~dB}, 13.8 \mathrm{~dB}$, and flatness of $0.5 \mathrm{~dB}, 0.6 \mathrm{~dB}$, $0.7 \mathrm{~dB}$, respectively. From Eq. (1), $f_{m}=20 \mathrm{GHz}$ with $\varphi_{2}=-1020 \mathrm{ps} / \mathrm{nm}$ dispersion results in a $163 \mathrm{ps}$ delay between adjacent comb lines, corresponds to a free-spectral-range (FSR) of $6.1 \mathrm{GHz}$. Thus, the first main passbands of the filters are centered at $6.1 \mathrm{GHz}$ for all cases, operating at the first Nyquist zone (DC to $10 \mathrm{GHz}$ ) free of spurious frequency [1,7]. The RF photonic filter responses are measured by vector network analyzer (VNA), shown in Fig. 2. (d), (e), and (f) for the three combs, all exhibit near perfect sinc response. Constant FSR is maintained for these filters, while the number of nulls and therefore the main passband $3 \mathrm{~dB}$ bandwidth, can be reconfigured from 960 $\mathrm{MHz}$ to $450 \mathrm{MHz}$ by varying the comb bandwidth from $120 \mathrm{GHz}$ to $240 \mathrm{GHz}$.

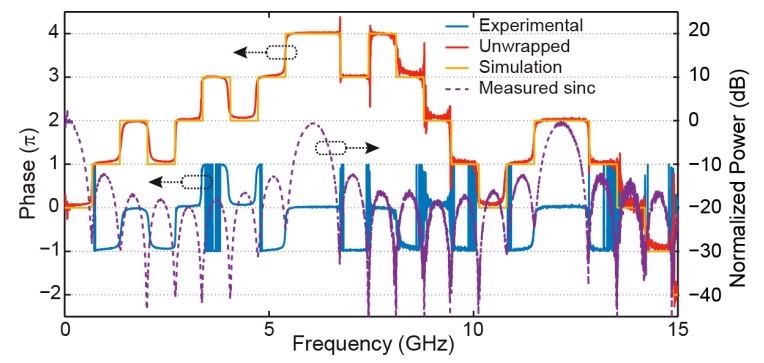

The retrieved phase response of the 9-line comb filter is shown in Fig. 3. Note that the quadratic phase is subtracted as the VNA is calibrated on single laser carrier. The measured unwrapped phase encounters $\pi$ phase jumps at every notch points of the amplitude response, as the sinc function flips the sign at every zero crossings. The measured phase response matches well with simulated pure $\pi$ phase jumps at amplitude nulls.

Fig. 3. Phase response of 9-line comb RF photonic filter.

We report versatile near perfect sinc shaped RF photonic filters obtained from filter-free optical comb. We show that the filter bandwidth is easily reconfigured by tuning the DC bias and RF power to the MZMs. Narrower filters can be reached by nonlinear expansion of the comb [9] while tuning of the FSR can be obtained by adjusting $f_{l}$ and $f_{2}$. This work was supported by the Swiss National Science Foundation under Grant 159897 and the Hasler Foundation.

[1] E. Hamidi, et al., "Tunable programmable microwave photonic filters based on an optical frequency comb," Trans. Microw. Theory Techn. 58, 3269-3278 (2010)

[2] V. R. Supradeepa, et al., "Comb based radiofrequency photonic filters with rapid tunability and high selectivity," Nat. Photon. 6, 186-194, (2012).

[3] J. Yao, "Microwave photonics," J. Lightw. Technol. 27, 314-335 (2009).

[4] M. Song, et al., "Reconfigurable and tunable flat-top microwave photonic filters utilizing optical frequency combs," Photon. Technol. Lett. 23, 1618-1620 (2011).

[5] Z. Zhu, et al., "All-positive-coefficient microwave photonic filter with rectangular response," Opt. Lett. 42, 3012-3015 (2017).

[6] M. A. Soto, et al. "Optical sinc-shaped Nyquist pulses of exceptional quality," Nat. Commun. 4, 2898 (2013).

[7] J. Liao, et al., "A spurious frequencies suppression method for optical frequency comb based microwave photonic filter," Laser Photon. Rev. 7, 4 (2013).

[8] X. Zhu, et al. "Novel programmable microwave photonic filter with arbitrary filtering shape and linear phase," Opt. Express 25, $9232-9243$ (2017).

[9] S. Cordette, et al., "Bandwidth and repetition rate programmable Nyquist sinc-shaped pulse train source based on intensity modulators and four-wave mixing," Opt. Lett. 39, 6668-6671 (2014). 